\title{
Identifying Remote Halo Giants in High-Latitude Fields with Kepler 2
}

\author{
Ruth C. Peterson
}

\author{
SETI Institute, 189 N Bernardo Ave, Mountain View, CA 94043
}

\begin{abstract}
This work sketches how SDSS ugr colors and Kepler 2 in halo fields can identify red giants $50-100 \mathrm{kpc}$ distant with minimal metallicity bias. For these mildly-reddened, metalpoor giants, $(g-r)$ o yields the effective temperature $T_{\text {eff }}$ to $100 \mathrm{~K}$. K2 can detect the p-mode oscillations of red giants and measure their frequency of maximum power $\nu_{\max }$. This sets the luminosity $L_{\mathrm{bol}}$ and thus the distance, plus an estimate of metallicity $[\mathrm{Fe} / \mathrm{H}]$.
\end{abstract}

Keywords. Galaxy: halo, Galaxy: stellar content, stars: abundances, stars: atmospheres, stars: distances, stars: Population II, techniques: photometric

Red giants are the best tracers of the remote halo. At their high luminosities they remain visible to $100 \mathrm{kpc}$, and their presence at all metallicities and ages encourages an unbiased sample of the stellar population. Here we note how Kepler (K2) and SDSS can find them.

In K2 fields in the Galactic halo, reddening is low, so the effective temperature $T_{\text {eff }}$ of a star known to be a metal-poor giant can be determined from its dereddened $(g-r)_{\mathrm{o}}$ color alone. Figure 1 shows the minimal spread in $(g-r)_{\mathrm{o}}$ at a given $T_{\text {eff }}$ at various metallicities $[\mathrm{Fe} / \mathrm{H}]$, for observed metal-poor giants and for the model atmospheres of Castelli \& Kurucz (2003) (CK03).

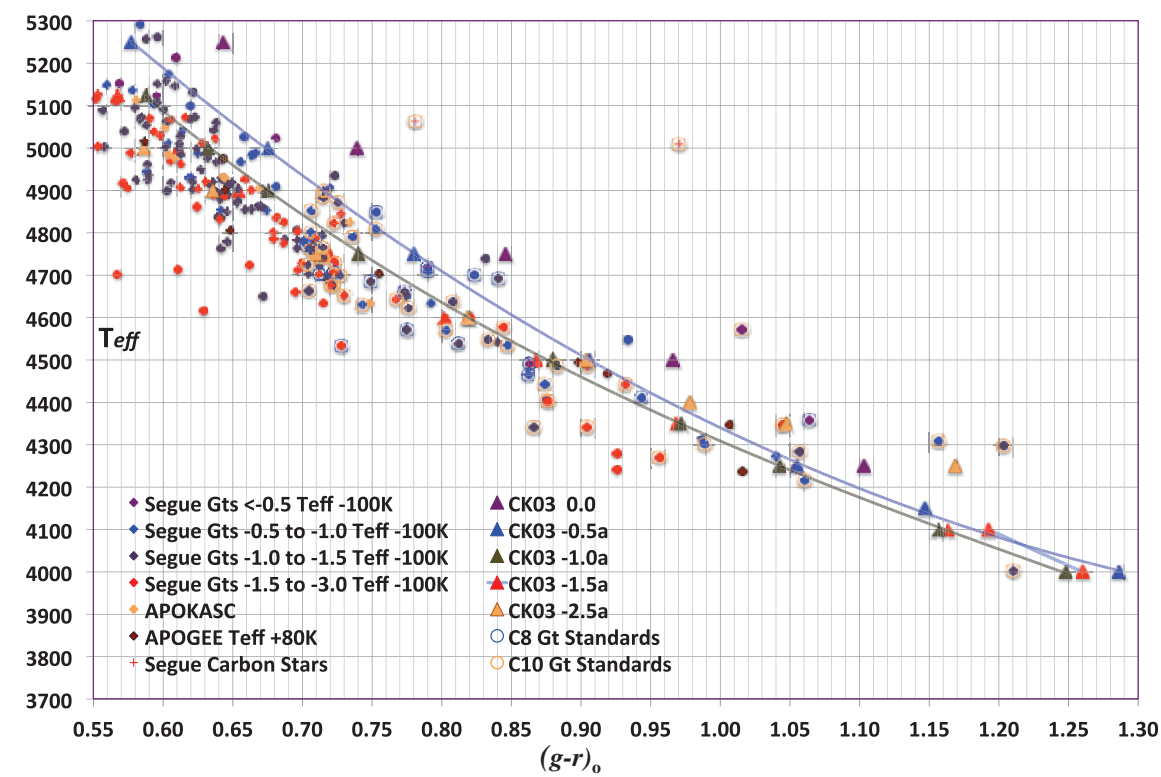

Figure 1. Temperatures from stellar SEGUE and APOGEE spectra (diamonds and circles) and CK03 giant models (triangles) are plotted vs. $(g-r)_{\mathrm{o}}$. The model $T_{\text {eff }}$ and $\log g$ values, from Yong et al. $(2013)$ at $[\mathrm{Fe} / \mathrm{H}]=1.6$, are $5125 \mathrm{~K} / 2.75,4900 \mathrm{~K} / 2.30,4750 \mathrm{~K} / 2.00,4600 \mathrm{~K} / 1.60$, $4500 \mathrm{~K} / 1.30,4350 \mathrm{~K} / 1.00$, and $4250 \mathrm{~K} / 0.75$. 


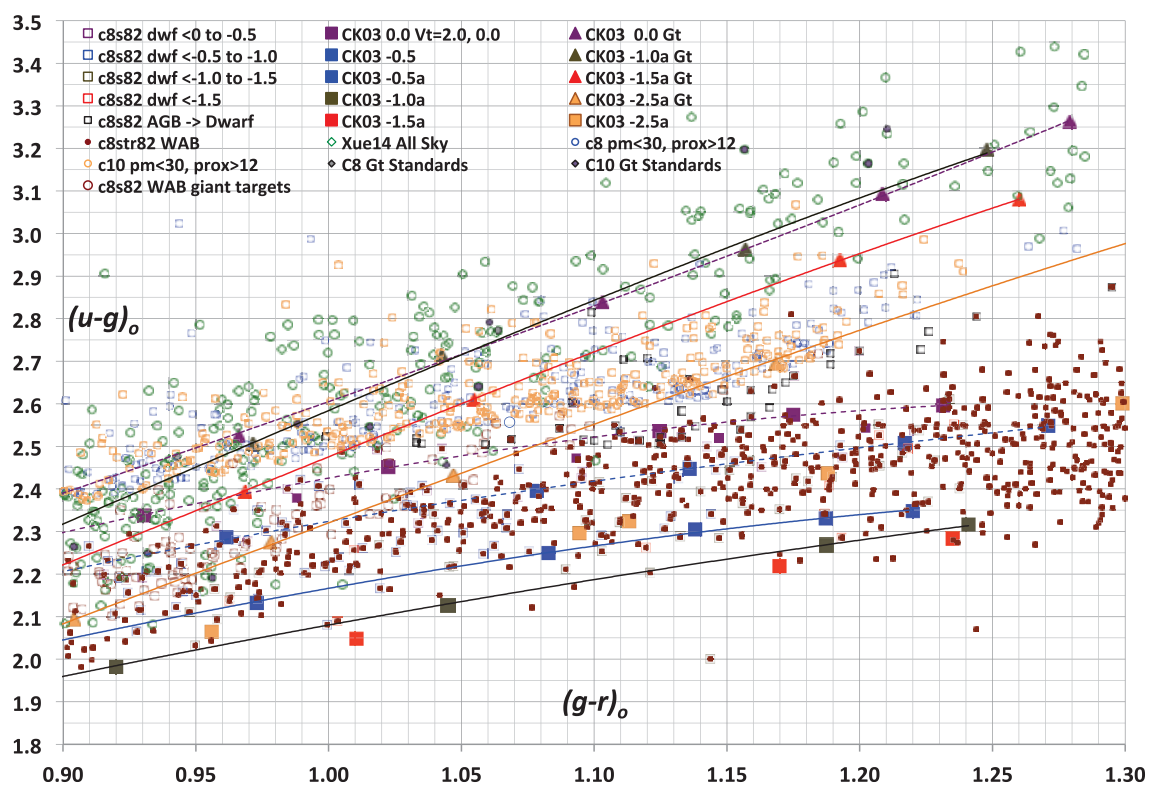

Figure 2. The dereddened colors of stars known to be giants (diamonds) or dwarfs (squares) are compared to the model colors of giants (triangles) and dwarfs (filled squares). Open circles are the dereddened colors for our proposed targets. Dark maroon circles are Bhatti et al. 2010 (WAB) stars, whose photometry is more precise. Note the paucity of cool giant WAB targets, hence their selection from the entire $\mathrm{C} 8$ and $\mathrm{C} 10$ fields.

K2 can identify remote giants asteroseismologically by detecting their p-mode oscillations. Those of dwarfs peak at frequencies too high to detect in long-cadence light curves, but those of cool, metal-poor giants fall within range, peaking at $\nu_{\max } \sim 200-$ $2 \mu \mathrm{Hz}$. Despite roll corrections, noise trials suggest K2 light curves should detect p-mode oscillations to Kepler magnitudes $K_{p} \sim 16.5$ for the most luminous metal-poor giants, reaching stars $100 \mathrm{kpc}$ away.

In Figure 2 we show K2 targets we have proposed in the halo fields C8 and C10 (open circles). Their dereddened $(u-g)_{\mathrm{o}}$ and $(g-r)_{\mathrm{o}}$ colors are those of cool giants, as seen from model CK03 colors (with $u-g$ increased by 0.2 ) of giants (diamonds) and dwarfs (squares), and observed colors of C8 and C10 SEGUE/APOGEE giants (filled diamonds) and the all-sky Xue et al. (2014) giants (open diamonds). Giants are scarce: the highquality Bhatti et al. (2010) (WAB) photometry (small filled circles) of Stripe 82 within C8 shows that foreground dwarfs dominate.

Applying this color selection to all SDSS + K2 halo fields should identify $\sim 100$ new giants. Set by stellar gravity, $\nu_{\max }$, plus $T_{\text {eff }}$ from $(g-r)_{\mathrm{o}}$, fix the giant luminosity $L_{\mathrm{bol}}$. This sets its distance, and also yields an estimate of $[\mathrm{Fe} / \mathrm{H}]$, since giant luminosity also depends on stellar metallicity (Xue et al. 2014). Thus SDSS + K2 alone can characterize the remote halo population.

\section{References}

Bhatti, W. A., Richmond, M. W., Ford, H. C., et al. 2010, Ap. J. Supp., 186, 233

Castelli, F. \& Kurucz, R. L. 2003, IAU Symp. No. 210, Modeling of Stellar Atmospheres, eds. N. Piskunov et al., CD-ROM poster A20; also astro-ph 0405087

Xue, X.-X., Ma, Z., Rix, H.-W., et al. 2014, Ap. J., 784, 170

Yong, D., Meléndez, J., Grundahl, F., et al. 2013, MNRAS, 434, 3542 